\title{
Challenges of AGB modelling with dust
}

\author{
Paolo Ventura ${ }^{1}$, Flavia Dell'Agli ${ }^{1,2}$, Marcella Di Criscienzo ${ }^{1}$, \\ D. A. García-Hernández ${ }^{3,4}$, Letizia Stanghellini ${ }^{5}$ and Raffaella \\ Schneider $^{1}$ \\ ${ }^{1}$ INAF - Osservatorio Astronomico di Roma, 00077, Monte Porzio Catone (RM), Italy \\ ${ }^{2}$ Università di Roma "La Sapienza", Piazzale Aldo Moro 5, 00136, Roma, Italy \\ ${ }^{3}$ Instituto de Astrofísica de Canarias, E-38205 La Laguna, Tenerife, Spain \\ ${ }^{4}$ Departamento de Astrofísica, Universidad de La Laguna (ULL), E-38206 La Laguna, \\ Tenerife, Spain \\ ${ }^{5}$ National Optical Astronomy Observatory, 950 N. Cherry Avenue, Tucson (AZ) 85719, USA
}

\begin{abstract}
We report on the most recent progresses on the modelling of the asymptotic giant branch (AGB) phase. We show that dust formation in the wind of AGBs is sensitive to the mass of the precursor, low-mass AGB with mass below $\sim 3 M_{\odot}$ producing mainly carbonaceous dust, whereas their counterparts of higher mass produce silicates and alumina dust. We discuss the uncertainty of the results, due to the poor knowledge of physical mechanisms highly important for AGB evolution, primarily convection and mass loss. We show how the chemical composition of planetary nebulae can be used to draw information on the main features of AGB evolution.
\end{abstract}

Keywords. stars: AGB and post-AGB, stars: evolution, planetary nebulae: general

\section{Introduction}

The modelling of the asymptotic giant branch phase has made significant progresses in the last years, with the introduction of the description of dust formation in the stellar wind (Ferrarotti \& Gail 2006). Such an improvement was required to interpret infrared (IR) data of individual AGBs provided by the various dedicated space missions, and to estimate the contribution from this class of objects to the overall dust pollution of the interstellar medium. The latter topic proves important for a series of still open problems, such as the understanding of the dust content of high-redshift quasars (Valiante et al. 2009) and the role played by AGBs in the formation and evolution of galaxies (Santini et al. 2014).

Dust production is strongly interfaced with the physical and chemical details of the AGB evolution, particularly the surface temperature, luminosity and surface chemical composition (Ferrarotti \& Gail 2006). The latter can be potentially altered by hot bottom burning (HBB) and third dregde-up (TDU). Unfortunately, the efficiency and the relative importance of these two mechanisms is far from being fully understood, as witnessed by the differences among the results obtained by various research groups involved in these studies (Karakas \& Lattanzio 2014). This clearly reflects on the results concerning dust production, which are further influenced by the uncertainties affecting the thermodynamical description of the wind (Ventura et al. 2014).

Based on these arguments, we understand that the comparison with the observations is currently the only possibility of extending our knowledge of the AGB phase. Dust production modelling turns out to be crucial to this aim, because it allows comparing the theoretical expectations with the several IR data available. 


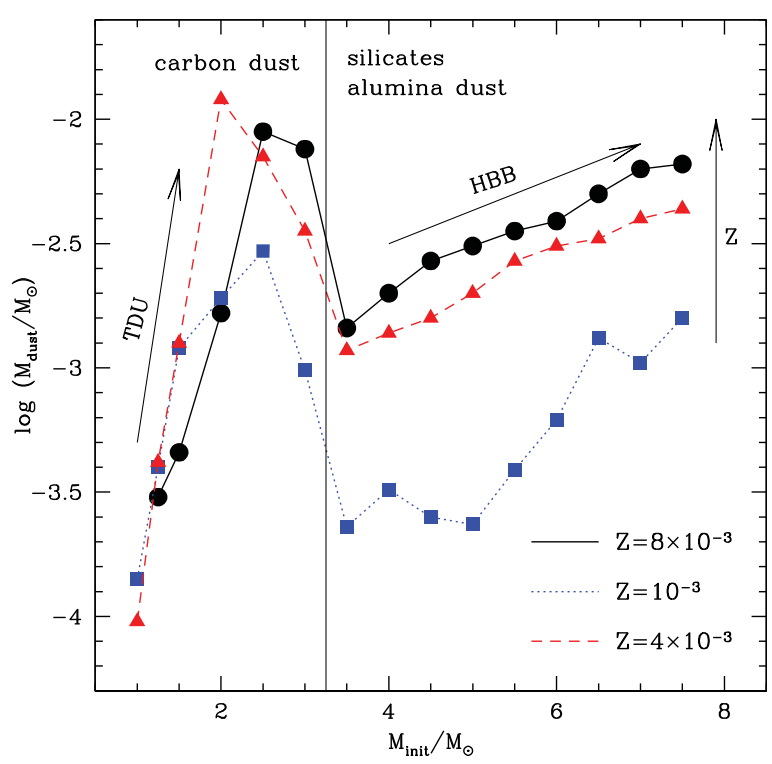

Figure 1. The dust mass produced by AGB stars as a function of the initial mass, for the metallicities $Z=10^{-3}, 4 \times 10^{-3}$ and $8 \times 10^{-3}$. The vertical line separates low-mass AGBs, producing mainly carbonaceous dust, from the higher mass counterparts, surrounded by silicates and alumina dust.

In this contribution, after a brief review of the main points concerning AGB evolution and the dust formation process, we address how the analysis of the chemical composition of planetary nebulae $(\mathrm{PNe})$ can provide an important contribution towards a full understanding of this still highly uncertain evolutionary phase.

\section{An overview of the AGB evolution properties}

All the stars with initial mass in the range $1 M_{\odot}<M_{\text {init }}<8 M_{\odot}$ evolve through the asymptotic giant branch phase (Iben 1982) $\dagger$. AGB stars are composed of a degenerate core, made up of carbon and oxygen (mass below $\sim 6-6.5 M_{\odot}$ ) or oxygen and neon $\left(M_{\text {init }} \geqslant 6.5 M_{\odot}\right)$, and a convective envelope, which is gradually lost by stellar winds. Once the external mantle is consumed, these stars evolve to become post-AGB stars and possibly $\mathrm{PNe}$, if the ejected envelopes have sufficient time to become ionized by the hot central stars before dissipating into the interstellar medium.

Shell CNO burning provides for most of the time the entire energy supply. Periodically, this H-burning layer is extinguished, when the ignition of a helium-rich buffer below occurs; because the latter nuclear activity takes place under conditions of thermal instability (Schwarzschild \& Harm 1965), it is commonly referred to as thermal pulse.

The AGB evolution is driven by the mass of the degenerate core, $M_{C}$ : the higher is $M_{C}$, the more efficient is $\mathrm{H}$-burning, the higher is the luminosity of the star (Paczyński 1970). Because the $M_{\text {init }}-M_{C}$ relationship is monotonically increasing, the stars of higher mass will evolve at larger luminosities and lose mass at a higher rate.

On the chemical side, the surface chemical composition can be altered by third dredge up and hot bottom burning. The former mechanism consists in the inwards penetration of the base of the convective mantle to reach regions previously touched by $3 \alpha$ nucleosyn-

$\dagger$ We will indicate with $M_{\text {init }}$ the mass of the star at the beginning of the evolution, whereas the current mass of the star will be indicated with $M$. 
thesis; the outcome of this process is a progressive enrichment in carbon of the surface regions. Hot bottom burning consists in the proton capture nucleosynthesis, activated at the bottom of the convective envelope, when the temperatures exceed $\sim 40 \mathrm{MK}$; the main effect of $\mathrm{HBB}$ is the depletion of the surface carbon in favour of nitrogen. Ignition of HBB requires core mass $M_{C} \sim 0.8 M_{\odot}$, which translates into initial masses $M_{\text {init }}>3 M_{\odot}$.

Based on the above arguments, we understand that the change in the surface chemistry of AGB stars depends critically on the initial mass. In low-mass AGBs the only active mechanism operating is TDU: repeated TDU episodes eventually turn oxygen-rich AGBs into carbon stars; this occurs for the stars with mass in the range $1.25 M_{\odot} \leqslant M_{\text {init }} \leqslant$ $3 M_{\odot}$. For stars of higher mass the modality with which the surface chemistry changes is still debated (Ventura \& D'Antona 2005): depending on the description of the convective instability, we find a variety of results, ranging from models exhibiting a pure HBB chemistry (thus reflecting the equilibria of proton-capture nucleosynthesis) to models in which TDU is still the dominant mechanism.

\section{Dust formation in the winds of AGB stars}

The dust formation mechanism is based on the schematisation set up by the Heidelberg group (Ferrarotti \& Gail 2006): the wind is assumed to expand isotropically from the surface of the star, under the effects of gravity, pressure gradient and radiation pressure, acting on the newly formed dust particles. In carbon-rich environments we consider formation of solid carbon, silicon carbide and solid iron, whereas in the winds of M-stars we account for the formation of silicates, alumina dust and solid iron.

The dust mass produced by AGB stars of different initial mass and metallicity is shown in Fig. 1. The amount of carbon dust produced by AGBs of mass below $\sim 3 M_{\odot}$ is independent of metallicity, because the carbon transported to the surface by TDU is of primary origin, thus not sensitive to Z; carbon stars of higher mass produce more dust, because they experience more TDU events, thus they reach a higher content of carbon during the AGB evolution (Ventura et al. 2014). AGBs with $M_{\text {init }}>3 M_{\odot}$ never become carbon stars, because the surface carbon is destroyed by HBB: consequently, these stars produce silicates and alumina dust. In this case the results are sensitive to the metallicity, because in higher $\mathrm{Z}$ models more silicon and aluminium are available to dust formation. The trend with mass is increasing, because higher $M_{\text {init }}$ models, evolving on bigger cores, experience stronger HBB and lose mass at a higher rate; this has the effects of rising the density of the wind, with more gas molecules available to form dust.

\section{The need of support from the observations: PNe chemistry}

The results described in the previous section, shown in Fig. 1, are significantly different from those presented by other research groups involved in these studies (see the discussion in Ventura et al. 2014). The dichotomy in the dust distribution among carbon dust (low-mass AGBs) and silicates (progenitors with $M_{\text {init }}>3 M_{\odot}$ ) holds as far as the convection model adopted is highly efficient, to allow strong HBB, as in our case; changing the description of convection results in a softer HBB (Ventura \& D'Antona 2005), thus making even massive AGBs to become carbon stars and form carbonaceous particles. The theoretical predictions are highly uncertain, both on the qualitative (kind of dust formed) and quantitative (dust mass produced) side (Ventura et al. 2014).

Because we are still far from self-consistent theories of convection and mass loss, the comparison with the observational evidence is currently the only possibility to allow meaningful step forwards towards a more reliable description of the AGB phase. 


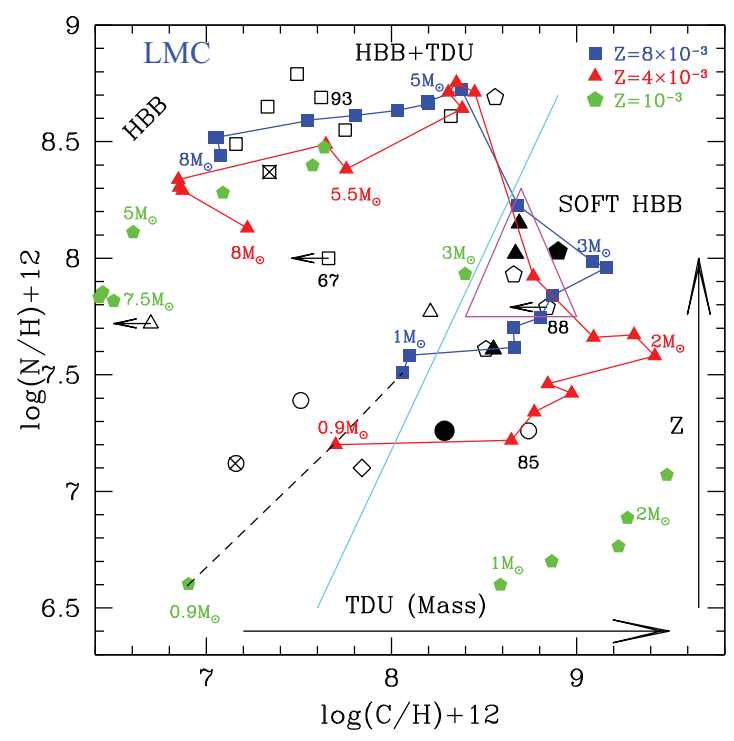

Figure 2. The chemical composition of PNe in the LMC from Leisy \& Dennefeld (2006) are shown as open, black points in the $\mathrm{C}$ vs. $\mathrm{N}$ plane. Squares, circles, triangles, pentagons and diamonds indicates PNe with, respectively, bipolar, round, bipolar core, elliptical and uncertain morphology (Stanghellini et al. 2000). The data symbols are filled where carbon dust, crossed where oxygen dust, has been detected (Stanghellini et al. 2007). The final chemical composition of AGB models of different mass and metallicity are indicated with full squares $\left(Z=8 \times 10^{-3}\right)$, triangles $\left(Z=4 \times 10^{-3}\right)$ and pentagons $\left(Z=10^{-3}\right)$. The dashed line indicates the chemistry expected after the effects of the first dredge-up, whereas the solid line delimits the border between carbon-rich and oxygen-rich chemistries.

PNe are the ideal targets for this scope. Their importance is related to the surface chemical composition, resulting from the combined effects of TDU and HBB in changing the relative distribution of the various species. Unlike the observation of individual AGBs, which offer a view of a specific evolutionary stage, the determination of the chemical composition of $\mathrm{PNe}$ presents a wider picture of the effects of the mechanisms able to alter the surface chemistry during the whole AGB phase.

Fig. 2 shows results from Ventura et al. (2015), where the PNe sample of the Large Magellanic Cloud (LMC) is used to deduce information on the evolution of AGBs, by comparing the observed chemistry of PNe with the final abundances predicted, at the very latest evolutionary phases. We show the abundances of carbon and nitrogen, the elements more sensitive to HBB and TDU: specifically, carbon is destroyed by HBB and produced by TDU, whereas nitrogen is synthesised at the base of the convective envelope when HBB is activated. The comparison among the models and the observations allows us drawing interesting conclusions.

The stars in the top, left region of the $\mathrm{CN}$ plane, with low $\mathrm{C}$ and high $\mathrm{N}$, show up a pure HBB nucleosynthesis; according to our modelling, this is only achieved in the AGBs of highest mass (above $\sim 6 M_{\odot}$ ), formed $40-80 \mathrm{Myr}$ ago. This finding provides an interesting confirmation that the effects of TDU can be null is some AGBs, possibly because the strong HBB favours a rapid loss of the external mantle, with little opportunity for TDU to operate.

$\mathrm{PNe}$ in the high $\mathrm{C}$, high $\mathrm{N}$ region experienced both TDU and HBB. They are likely descendants from AGBs with $3 M_{\odot}<M_{\text {init }}<6 M_{\odot}$, younger than $\sim 200 \mathrm{Myr}$. These stars suffer HBB from the early AGB phases, with the depletion of the surface carbon; 
in the final evolutionary stages, when HBB is extinguished, TDU takes over, increasing the surface carbon.

The stars within the triangle in Fig. 2 have a high carbon and show up a modest increase in nitrogen, a signature of a mild HBB, active only during a few interpulses. These objects descend from stars with $M_{\text {init }} \sim 3-3.5 M_{\odot}$, which experience HBB, before the gradual increase in the surface carbon eventually leads to a general cooling of the structure, that turns off HBB.

$\mathrm{PNe}$ below the afore mentioned triangle, on the right of the diagonal, cyan line, suffered several TDU episodes, which turned them into carbon stars. No signature of HBB is evident. According to our modelling, these are older objects (age above $500 \mathrm{Myr}$ ), descending from stars with $M_{\text {init }}<3 M_{\odot}$. The highest carbon observed can be used to set a limit to the efficiency of TDU.

\section{Conclusions}

We present the most recent results concerning dust formation in the wind of AGB stars of various initial mass and metallicity. The dust formed exhibits a dichotomy, with lowmass AGBs producing mainly carbonaceous dust, whereas their counterparts of higher mass produce exclusively silicates and alumina dust. This is strongly interfaced with the relative weight of the mechanisms acting in changing the surface chemical composition, namely third dregde up and hot bottom burning.

These results are highly uncertain, as they depend critically on the physical ingredients used, primarily convection and mass loss modelling. This makes the comparison with the observational evidence a crucial requirement for the development of these studies.

The observation of the chemical composition of PNe proves a very promising field to be investigated. The chemistry of these objects is determined by a sequence of events taking place during the whole AGB phase, thus allowing understanding the relative importance of the various mechanisms able to alter the relative abundances of the various chemical species.

\section{References}

Ferrarotti, A. D. \& Gail, H. P. 2006, A\& $A, 553,576$

Iben, I., Jr. 1982, ApJ, 260, 821

Karakas, A. I. \& Lattanzio, J. C. 2014, PASA, 31, e030

Leisy, P. \& Dennefeld, M. 2006, A\& $A, 456,451$

Paczyński, B. 1970, Acta Astr., 20, 47

Santini, P., Maiolino, R., Magnelli, B., et al. 2014, A\& A, 562, A30

Schwarzschild, M. \& Harm, R. 1965, ApJ, 142, 855

Stanghellini, L., Shaw, R. A., Balick, B., \& Blades, J. C. 2000, ApJL, 534, L167

Stanghellini, L., Garcia-Lario, P., \& García-Hernández, D. A. et al. 2007, ApJ, 671, 1669

Valiante, R., Schneider, R., Bianchi, S., Andersen, A., \& Anja, C. 2009, MNRAS, 397, 1661

Ventura, P. \& D'Antona F. 2005, A\&A A, 431, 279

Ventura, P., Dell'Agli, F., Di Criscienzo, M., Schneider, R., Rossi, C., La Franca, F., Gallerani, S., \& Valiante R. 2014, MNRAS, 439, 977

Ventura, P., Stanghellini, L., Dell'Agli, F., García-Hernández, D. A. \& Di Criscienzo, M. 2015, MNRAS, 452, 3679 